\title{
A Logic for Synchronous Transitions with Dynamic Conflict Resolution
}

\author{
Vanderlei Moraes Rodrigues* Flávio Rech Wagner \\ Instituto de Informática, UFRGS ${ }^{\dagger}$ \\ \{vandi,flavio\}@inf.ufrgs.br
}

\begin{abstract}
This paper introduces a formalism named DSYNC aimed at the design and verification of synchronous concurrent systems. The components of this formalism are a transition system and a first-order linear-time temporal logic. The DSY NC transition system adopts a synchronous computation model, includes a method to solve write-conflicts, and represents transitions as possibly non-terminating imperative commands. The conflict resolution method is dynamic because it detects conflicts at run-time. The DSYNC logic allows for formal reasoning about DSYNC transition systems using compositional and modular proofs. Such features are missing in other formalisms based on transition systems and temporal logics, although they are important for the verification of a large class of systems. This paper also discusses some of the pragmatics in verifying systems with DSYNC, and considers some extensions to the formalism. DSYNC is based on the Hoare logic and the UNITY formalism.
\end{abstract}

Keywords: linear-time temporal logic, Hoare logic, UNITY, synchronous systems.

\section{Introduction}

The class of formalisms composed of a transition system and a first-order linear-time temporal logic includes the Manna and Pnueli logic [17, 18], TLA [16], UNITY [8, 20], ST [30], and other formalisms [26, 29]. Due to their expressiveness and flexibility, they have been successfully employed in the description and verification of concurrent or reactive systems in several application fields. However, these formalisms deal with asynchronous systems mostly. A distinct class of computational systems is that of synchronous systems. It includes programming languages such as Esterel [2], hardware description languages such as VHDL [22], and specification formalisms such as evolving algebras $[12,13,14]$. Works on the verification of such systems either explore restricted techniques such as finite model-checkers, or depend on idiosyncrasies of a particular notation, or are not mature yet.

*Partially supported by QaP-For/FAPERGS.

${ }^{\dagger}$ Caixa postal 15064. 91501-970, Porto Alegre, Brazil. Fax +55(51)319-1576. 
This paper introduces a formalism named DSYNC aimed at the description and verification of synchronous systems using a transition system and a first-order lineartime temporal logic. The proposed synchronous computation model includes a conflict resolution method detecting conflicts dynamically, during system execution. DSYNC also features a representation for transitions as (possibly non-terminating) imperative commands, a modular and compositional approach to proof development, and a good catalog of elementary verification techniques. There are extensions to DSYNC dealing with generic parameters, regular structures, and statically detected conflicts.

We believe the development of DSYNC is a contribution to the application of firstorder linear-time temporal logics on the verification of reactive systems. The main formalisms in this class listed above (UNITY, TLA, etc.) do not handle synchronous systems, dynamic resolution of write-conflicts, and other features of DSYNC. These features are essential for the verification of VHDL and evolving algebras, for instance, but they are not addressed appropriately in earlier formalisms.

We also believe DSYNC contributes to the work on the verification of synchronous systems. Most works on this field concentrate on finite model-checkers [9]. This technique is quite effective, but it does not handle some important situations. It does not allow for modular proofs, where properties of a system are derived from properties of its components only, without any knowledge on the actual definition of components. It does not solve conflicts dynamically, and it is not appropriate for parametric and regular systems either, because such systems may generate large or infinite sets of states. Other works employ more general formalisms, but most of them deal with restricted systems or result in complex formalisms which are hard to apply in the verification of actual systems [7, 28]. DSYNC is quite general, yet easy to use.

Work on DSYNC started as a wish to apply the UNITY logic in the verification of VHDL designs. While adapting UNITY to the semantics of VHDL, we came up with a formalism embodying a general model of synchronous computation with dynamic conflict resolution. The same model and verification techniques apply to other hardware description languages, to synchronous programming languages, and to some specification formalisms such as evolving algebras. We describe elsewhere the practical work aimed specifically at VHDL [24]. This paper describes DSYNC as a general formalism for the verification of synchronous systems, develops some foundation work, and discusses important description and proof techniques for DSYNC. The resulting formalism is quite general and flexible, although it cannot reach the automation level of finite modelcheckers.

This paper is organized as follows. Section 2 discusses the synchronous computation model and introduces the DSYNC transition system, and section 3 presents the DSYNC logic. Section 4 studies some methods to apply this formalism in the specification and verification of synchronous systems. Section 5 comments on related works, and the last section presents some concluding remarks.

\section{Transition System}

The components of a transition system are a set of variables and a set of transitions. They represent the (possibly infinite) set of system states and the permitted 


$$
\begin{aligned}
& t_{a} \rrbracket t_{b}: \begin{array}{l}
a: \\
b: 0 \\
b: 0
\end{array} \rightarrow \begin{array}{|l|}
\hline 1 \\
\hline 0
\end{array} \rightarrow \begin{array}{|l|}
\hline 2 \\
\hline 0
\end{array} \rightarrow \begin{array}{l}
2 \\
\hline 1
\end{array} \rightarrow \begin{array}{l}
3 \\
\hline 1
\end{array} \rightarrow \cdots
\end{aligned}
$$

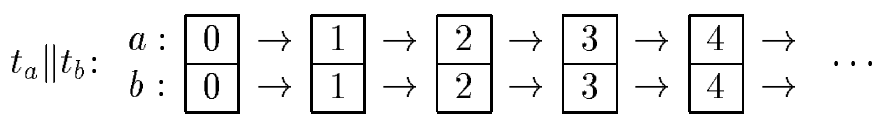

Figure 1: Asynchronous and synchronous computation models

state changes. According to the asynchronous computation model adopted by UNITY, TLA, and most transitions systems, each computation step non-deterministically selects and runs exactly one elementary transition. Let $t_{a}$ and $t_{b}$ be the transitions $a:=a+1$ and $b:=b+1$ respectively. The left-half of Figure 1 shows a computation of the asynchronous combination $t_{a} \rrbracket t_{b}$. Distinctly from these formalisms, DSYNC follows a synchronous computation model, where each computation step runs each elementary transition once. The right-half of Figure 1 shows a computation of the synchronous combination $t_{a} \| t_{b}$.

Figure 2 presents the DSYNC transition system. A program is a pair $\langle b \mid t\rangle$, where the program body $t$ is a synchronous combination of a finite set of elementary transitions, and the initial condition $b$ is a boolean expression describing the initial value of variables. When the initial condition is irrelevant, we annotate program $\langle b \mid t\rangle$ as $t$ only. Standard imperative commands represent elementary transitions. For simplicity, we assume variables and expressions are defined as usual, expressions are total, and programs are well-typed. We also need some syntactical restriction on initial conditions to ensure they are consistent. In this paper, they are restricted to a conjunction of terms $x=k$, where $k$ is a constant.

A state $\sigma$ is a mapping of variable names to values, and $\widehat{\sigma}(e)$ denotes the value in $\sigma$ of an expression $e$ (or condition, or assertion). Notation $f: X \rightarrow Y$ indicates $f$ is a partial function mapping elements of $X$ to elements of $Y$, and $\left\{x_{1} \mapsto y_{1}, \ldots, x_{n} \mapsto y_{n}\right\}$ represents a finite mapping of $x_{i}$ to $y_{i}$. When $f$ is undefined on $x$, we write $f(x)=\perp$. Notation $f \downarrow Z$ denotes $f$ with its domain restricted to a set $Z$ of variables names, and $f \oplus g$ denotes $f$ updated with function $g$ :

$$
(f \downarrow Z)(x)=\left\{\begin{array}{ll}
f(x) & \text { when } x \in Z \\
\perp & \text { otherwise }
\end{array} \quad(f \oplus g)(x)= \begin{cases}g(x) & \text { when } g(x) \neq \perp \\
f(x) & \text { otherwise }\end{cases}\right.
$$

The rules in Figure 2 present an operational semantics for the DSYNC transition system. The four-argument relations $s \mid \omega \triangleright \sigma \stackrel{\text { cmd }}{\longrightarrow} \sigma^{\prime}$ and $t \mid \omega \triangleright \sigma \stackrel{\text { trn }}{\longrightarrow} \sigma^{\prime}$ indicate that the execution of command $s$ or transition $t$ in a state $\sigma$ produces a state $\sigma^{\prime}$, assigning new values to the variables named in the write-set $\omega$. Write-sets are sets of variable names recording the assignments that the program performs. We need to build them along the computation because they depend on the initial state. For instance, if $x>0$ then $y:=x$ else skip does not always write to $y$.

Rules S1 to S7 define the command semantics as usual [1], except that they also collect the write-sets. S2 adds a variable name to the write-set, and the remaining rules only combine these sets. Rules $\mathrm{S} 8$ to $\mathrm{S} 10$ define a semantics for transitions. Rule S8 executes elementary transitions, beginning the command computation with an 


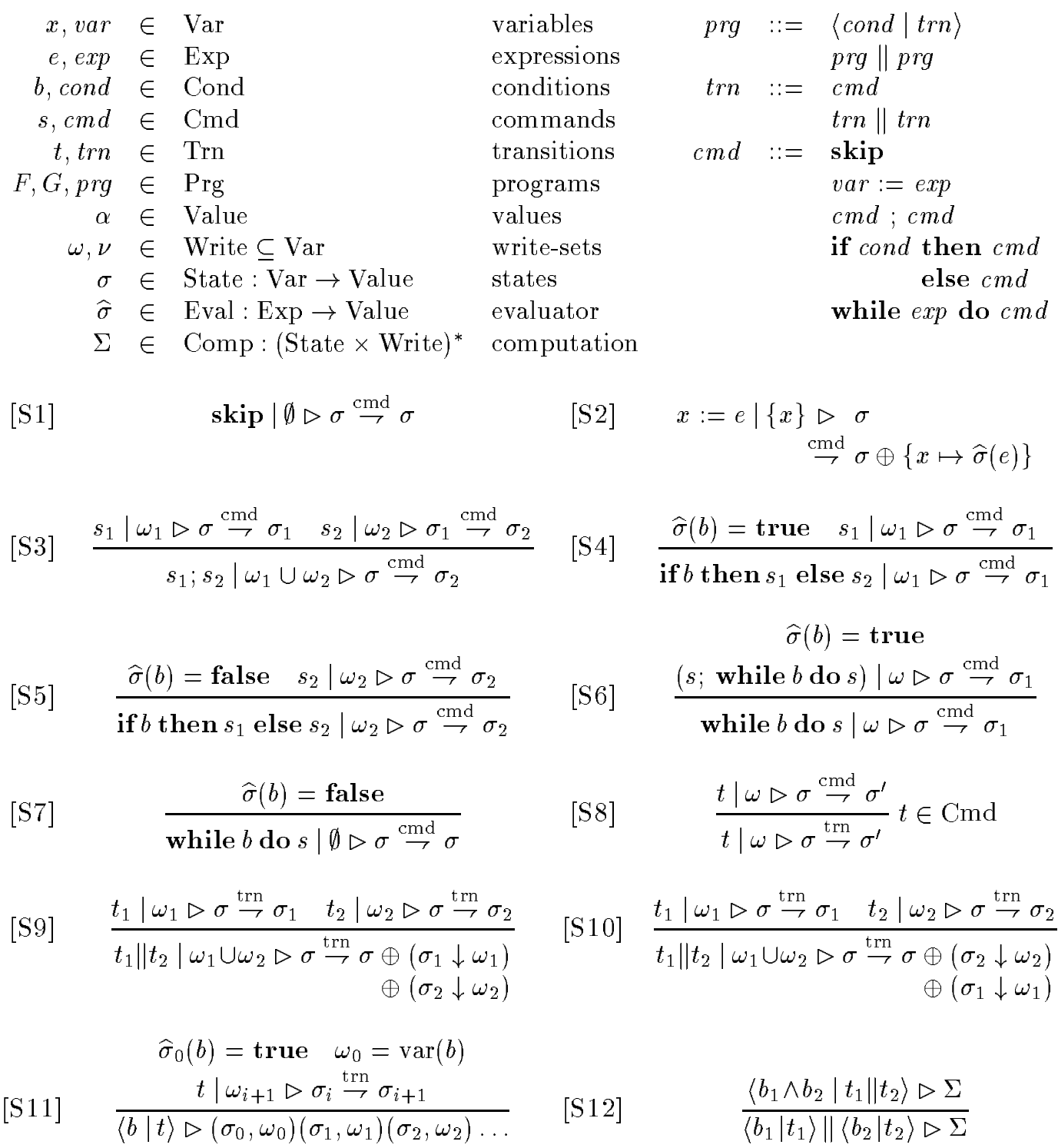

Figure 2: DSYNC transition system

empty write-set. Rules S9 and S10 describe the synchronous combination of transitions, including the dynamic method to solve write-conflicts.

Two transitions generate a write-conflict when they try to assign to the same variable at the same time. To account for this situation, we define the semantics for synchronous combinations as follows. To execute $t_{1} \| t_{2}$, we give a distinct copy of the initial state to each transition, execute them independently, and then update the initial state with the contribution of each transition. The contribution of a transition is the set of variables the transition writes while executing. The order we apply the contributions to the final state is not determined. Therefore, the last transition to apply its contribution will define the final value of conflicting variables, and the computation of a synchronous 

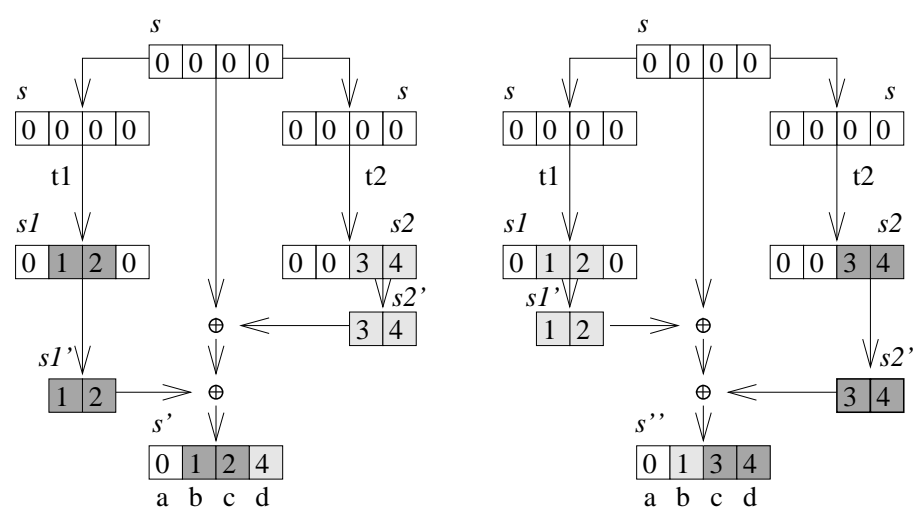

Figure 3: Execution of a synchronous combination

combination generating write-conflicts is non-deterministic. For instance, let $t_{1}$ and $t_{2}$ be $(b:=1 ; c:=2)$ and $(c:=3 ; d:=4)$. Figure 3 shows the two possible computations of $t_{1} \| t_{2}$, where $s^{\prime}$ and $s^{\prime \prime}$ are the final states.

Rules S9 and S10 describe the behavior above, where $\left(\sigma_{i} \downarrow \omega_{i}\right)$ is the contribution of transition $t_{i}$. These rules only differ in the order they apply these contributions, accounting for the non-deterministic conflict resolution method. This method is dynamic because the write-sets $\omega_{i}$ are built along the computation, ensuring a precise detection of conflicts. In [25], we explore a static conflict resolution method, where we assume a transition $t$ assigns to all variables on the left of the assignment occurring in $t$. This method is simpler than DSYNC, since we build this set of variables without looking at the actual transition computation. It is adequate to some classes of restricted systems, but it is pessimistic, because it may indicate a write-conflict when no one actually happens. The present method offers a more precise treatment for write-conflicts.

We chose the synchronous combinator semantics above for many reasons. It seems to generalize the semantics of several synchronous formalisms, such as VHDL and evolving algebras, it restricts the interaction between component transitions, and it represents the conflict resolution method explicitly as a single and well-defined operation (the state update operation). As a consequence, it is easier to reason about component transitions independently, and the synchronous combinator shows up several properties. For instance, these combinator is idempotent, commutative, and skip is its neutral element.

The last rules in Figure 2 define a binary relation $F \triangleright \Sigma$ indicating that program $F$ generates the computation $\Sigma=\left(\sigma_{0}, \omega_{0}\right)\left(\sigma_{1}, \omega_{1}\right)\left(\sigma_{2}, \omega_{2}\right) \ldots$, where each $\omega_{i}$ names the variables written during the computation of $\sigma_{i}$. In essence, what programs add to transitions is a description of the initial states. According to S11, a computation is a (usually infinite) sequence of states and write-sets generated through the repeated execution of the program body that starts in a state satisfying the initial program condition. To define the synchronous combination of programs, S12 just unfolds the program combination. 


\section{Logic}

We use the DSYNC logic to verify statements about a DSYNC transition system. It is derived from the Hoare logic [1] and the UNITY logic [8, 20]. The DSYNC logic employs modified Hoare triples in the form $\{p\} s \mid \omega\{q\}$ and $\{p \mid\} t \mid \omega\{q \mid\}$. They represent the statement "if the execution of command $s$ or transition $t$ begins in a state where $p$ holds, then it does terminate in a state where $q$ holds, and it may write to the variables in $\omega "$. Assertions $p$ and $q$ are formulas from standard predicate logic over state variables.

We employ distinct notations for triples over commands and transitions to emphasize that the computation of a transition does not include the intermediate states generated during the computation of commands. We need to add write-sets to triples because the rules for synchronous combinations depend on them, and they are built dynamically, along the actual computation. However, a triple may describe several computations with distinct write-sets. Therefore, the write-set of a triple can be larger then the actual write-set built along a computation.

Using the semantics presented in the previous section, we may precisely describe the modified Hoare triples. Let $\sigma$ be any state where $\widehat{\sigma}(p)=$ true. The triple $\{p\} s \mid \omega\{q\}$ holds if both conditions below are satisfied:

- there exists some $\sigma^{\prime}$ and $\omega^{\prime}$ where $\left(s \mid \omega^{\prime} \triangleright \sigma \stackrel{\text { cmd }}{\longrightarrow} \sigma^{\prime}\right)$, and $\widehat{\sigma}^{\prime}(q)=\operatorname{true}$, and $\omega^{\prime} \subseteq \omega$; - for all $\sigma^{\prime}$ and $\omega^{\prime}$ where $\left(s \mid \omega^{\prime} \triangleright \sigma \stackrel{\text { cmd }}{\longrightarrow} \sigma^{\prime}\right)$, we get $\widehat{\sigma}^{\prime}(q)=$ true, and $\omega^{\prime} \subseteq \omega$.

Transition triples are similarly defined.

Figure 4 lists the rules comprising the DSYNC logic. It is organized in three layers, reflecting the transition system organization. The bottom layer comprising rules A1 to A6 is the standard Hoare logic for total correctness of commands [1] extended to deal with write-sets. Rule A1 allows the enlargement of write-sets, A3 ensures the assigned variable is included in the write-set, and the remaining rules only carry these sets forward.

Rules B1 to B4 constitute the middle layer, allowing the verification of statements about transitions and their combinations. These rules define triples over transitions. B1 is an adaption (strengthening and weakening) rule, B2 describes elementary transitions, and rules B3 and B4 describe the synchronous combinator. To simplify the modular development of systems, rules for combinations need to be compositional. It means $\{p \mid\} t_{1} \| t_{2} \mid \omega\{q \mid\}$ must be derived from triples over $t_{1}$ and $t_{2}$ alone. However, the synchronous combination $t_{1} \| t_{2}$ does not preserve all triples over $t_{1}$ and $t_{2}$. For instance, let $t_{a}$ and $t_{b}$ be $a:=a+1$ and $b:=b+1$. In this case, $\{a=b\} t_{a} \mid \omega\{a \neq b \mid\}$ holds, but $\{a=b \mid\} t_{a}|| t_{b} \mid \omega\{a \neq b \mid\}$ does not. Actually, as Figure 1 shows, what does hold is $\{a=b \mid\} t_{a} \| t_{b} \mid \omega\{a=b \mid\}$.

To describe the synchronous combination, there are two cases to consider. First, $t_{1} \| t_{2}$ preserves a triple $\{p \mid\} t_{1} \mid \omega_{1}\{q\}$ over the component transition $t_{1}$ if the other component $t_{2}$ does not change any variables occurring in $q$. Rule B3 describes this case. The proviso in this rule uses the write-sets of each component transition to ensure the condition above for both components. Informally, this rule handles statements over variables that do not have a write-conflict. Second, $t_{1} \| t_{2}$ preserves a triple $\{p \mid\} t_{1} \mid \omega_{1}\{q \mid\}$ 
$[\mathrm{A} 1]$

$$
\frac{\{p \wedge b\} s_{1}\left|\omega\{q\} \quad\{p \wedge \neg b\} s_{2}\right| \omega\{q\}}{\{p\} \text { if } b \text { then } s_{1} \text { else } s_{2} \mid \omega\{q\}}
$$$$
\frac{p \Rightarrow p^{\prime} \quad\left\{\left|p^{\prime}\right|\right\} t \mid \omega^{\prime}\left\{\left|q^{\prime}\right|\right\} \quad \omega^{\prime} \subseteq \omega \quad q^{\prime} \Rightarrow q}{\{p \mid\} t \mid \omega\{|q|\}}
$$

$$
\frac{\left\{\left|p_{1}\right|\right\} t_{1}\left|\omega_{1}\left\{\left|q_{1}\right|\right\} \quad\left\{\left|p_{2}\right|\right\} t_{2}\right| \omega_{2}\left\{\left|q_{2}\right|\right\}}{\left\{\left|p_{1} \wedge p_{3}\right|\right\} t_{1}|| t_{2} \mid \omega_{1} \cup \omega_{2}\left\{q_{1} \wedge q_{2} \mid\right\}} \dagger
$$

$$
\frac{p \Rightarrow p^{\prime} \quad p^{\prime} \operatorname{co} q^{\prime} \text { in } F \mid \omega^{\prime} \begin{array}{l}
q^{\prime} \Rightarrow q \\
\omega^{\prime} \subseteq \omega
\end{array}}{p \operatorname{co} q \text { in } F \mid \omega}
$$

$$
\operatorname{inv} p \text { in } F \mid \omega \equiv \begin{aligned}
& (\text { init } p \text { in } F \mid \omega) \wedge \\
& (p \cos p \text { in } F \mid \omega)
\end{aligned}
$$$$
\operatorname{inv} r \text { in } F \mid \omega
$$$$
\frac{(p \wedge r) \operatorname{co}(q \wedge r) \text { in } F \mid \omega}{p \cos q \text { in } F \mid \omega}
$$$$
p \text { leads } p \text { in } F \mid \omega
$$

$$
\frac{\{|p|\} t \mid \omega\{q \mid\}}{p \operatorname{co} q \operatorname{in}\langle b \mid t\rangle \mid \omega}
$$

$$
\frac{\{|p|\} t_{1}\left|\omega_{1}\{|q|\} \quad\{|p|\} t_{2}\right| \omega_{2}\{|q|\}}{\{|p|\} t_{1}|| t_{2} \mid \omega_{1} \cup \omega_{2}\{q \mid\}} \ddagger
$$

$$
\frac{\operatorname{var}(b) \subseteq \omega}{\text { init } b \text { in }\langle b \mid t\rangle \mid \omega}
$$

$\frac{\operatorname{inv} r \text { in } F \mid \omega \quad p \operatorname{co} q \text { in } F \mid \omega}{(p \wedge r) \cos (q \wedge r) \text { in } F \mid \omega}$

$$
\begin{gathered}
p \Rightarrow p^{\prime} \quad p^{\prime} \text { leads } q^{\prime} \text { in } F \mid \omega^{\prime} \begin{array}{r}
q^{\prime} \Rightarrow q \\
\omega^{\prime} \subseteq \omega
\end{array} \\
\hline p \text { leads } q \text { in } F \mid \omega
\end{gathered}
$$

$$
\frac{p \operatorname{co} q \text { in } F \mid \omega}{p \text { leads } q \text { in } F \mid \omega}
$$

$p_{1}$ leads $q_{1}$ in $F \mid \omega_{1}$

$$
\frac{p_{2} \text { leads } q_{2} \text { in } F \mid \omega_{2}}{\left(p_{1} \vee p_{2}\right) \text { leads }\left(q_{1} \vee q_{2}\right) \text { in } F \mid \omega_{1} \cup \omega_{2}}
$$

$$
\left\langle b_{1} \mid t_{1}\right\rangle \|\left\langle b_{2} \mid t_{2}\right\rangle \equiv\left\langle b_{1} \wedge b_{2}\left|t_{1}\right| \mid t_{2}\right\rangle
$$

$$
\begin{aligned}
& \dagger \quad \operatorname{var}\left(q_{1}\right) \cap \omega_{2}=\emptyset \wedge \operatorname{var}\left(q_{2}\right) \cap \omega_{1}=\emptyset \\
& \ddagger \quad \operatorname{var}(q) \cap \omega_{1}=\operatorname{var}(q) \cap \omega_{2}
\end{aligned}
$$

Figure 4: DSYNC logic

if both component transitions change the same variables in $q$ and the triple holds for both component transitions separately. Informally, it means that there is a write-conflict, but both transitions have the same affect on the conflicting variables. Rule B4 describes this case. We observe that both rules depend on the precise description of the write-sets of each component transition.

The top layer in the DSYNC logic is a temporal logic dealing with statements about 


$$
\begin{array}{rll}
\text { init } p \text { in } F \mid \omega & \text { iff } & \Sigma_{0}(p) \wedge \Sigma_{0} \subseteq \omega \\
p \text { co } q \text { in } F \mid \omega & \text { iff } & \left(\forall i: \Sigma_{i}(p) \Rightarrow\left(\Sigma_{i+1}(q) \wedge \Sigma_{i+1} \subseteq \omega\right)\right) \\
\text { inv } p \text { in } F \mid \omega & \text { iff } & \left(\forall i: \Sigma_{i}(p) \wedge \Sigma_{i} \subseteq \omega\right) \\
p \text { leads } q \text { in } F \mid \omega & \text { iff } & \left(\forall i: \Sigma_{i}(p) \Rightarrow\left(\exists j: j \geq i \wedge \Sigma_{j}(q) \wedge\left(\forall k: i \leq k \leq j \Rightarrow \Sigma_{k} \subseteq \omega\right)\right)\right)
\end{array}
$$

Figure 5: Temporal properties

complete program computations. Formulas in this layer are called properties. They are built from a temporal connective applied to assertions, they are always attached to programs, and they cannot be nested. Figure 5 lists the properties and their meaning. The left column shows a property over a program $F$ writing to variables in $\omega$, and the right column shows a condition on the computations $\Sigma=\left(\sigma_{0}, \omega_{0}\right)\left(\sigma_{1}, \omega_{1}\right)\left(\sigma_{2}, \omega_{2}\right) \ldots$ generated by $F$. A property holds if all computations of $F$ satisfy the corresponding condition. In the right column, $\Sigma_{i}(p)$ means that there is a position $i$ in $\Sigma$ and assertion $p$ holds in the corresponding state, i.e., $\Sigma_{i} \neq \perp$ and $\widehat{\sigma}_{i}(p)=$ true. Likewise, $\Sigma_{i} \subseteq \omega$ means that position $i$ only writes to the variables in $\omega$, i.e., $\omega_{i} \subseteq \omega$.

Informally, properties may be read as follows: init $p$ in $F \mid \omega$ means " $p$ holds in the first state, and only the variables in $\omega$ are initialized", $p$ co $q$ in $F \mid \omega$ means "if $p$ holds, then $q$ holds in the next state, assigning to the variables in $\omega$ only", inv $p$ in $F \mid \omega$ means " $p$ holds in all states, and only the variables in $\omega$ are assigned", and $p$ leads $q$ in $F \mid \omega$ means "if $p$ holds, then $q$ will hold in some future state, meanwhile assigning to the variables in $\omega$ only".

It is implicit in the comments above that properties only consider the reachable states of a program $F$, i.e., the states generated through a computation of $F$. Depending on initial conditions, this set is smaller than the set of all possible states. This difference affects the statements we may prove. For instance, let $t_{b}$ be $b:=b+a$, and let $F_{b}$ be $\left\langle a=2 \mid t_{b}\right\rangle$. We cannot prove $\{\operatorname{even}(b)\} t_{b} \mid \omega\{\operatorname{even}(b)\}$. However, we may prove even $(b)$ co even $(b)$ in $F_{b} \mid \omega$ because $a$ always holds 2 in the computations of $F_{b}$. The difference results from the fact that the triple considers all pairs of initial and final states for $t_{b}$, while the property only considers the pairs of reachable states.

Rules $\mathrm{C} 1$ to $\mathrm{C} 12$ in Figure 4 define the temporal connectives. C1, C3, and $\mathrm{C} 8$ are adaption rules, $\mathrm{C} 2$ describes the initial states, $\mathrm{C} 4$ is the base case for co properties, and $\mathrm{C} 5$ defines temporal invariants. The substitution rules $\mathrm{C} 6$ and $\mathrm{C} 7$ allow for the introduction and elimination of temporal invariants in assertions. These two rules are the device that restricts properties to the set of reachable states. Rules C8 to C11 define the leads connective, and $\mathrm{C} 12$ describes program combinations.

The DSYNC temporal logic is derived from the UNITY logic. The link between these logics follows from the fact that a synchronous transition program corresponds to a UNITY program with a single (non-deterministic) transition. Since we consider these restricted UNITY programs only, some UNITY rules become simpler in DSYNC. When moving to DSYNC, we also review all property definitions to account for nondeterminism, non-terminating transitions, and write-sets. Additionally, DSYNC omits an infinitary UNIT Y rule for leads properties, because all DSYNC programs are finite.

As a design decision, DSYNC omits the skip steps (stuttering steps [16]) in the definition of co. We believe they are not necessary in a synchronous transition system 


$$
\begin{aligned}
& \text { [D1] } \frac{\left\{p_{1}\right\} s\left|\omega\left\{q_{1}\right\} \quad\left\{p_{2}\right\} s\right| \omega\left\{q_{2}\right\}}{\left\{p_{1} \vee p_{2}\right\} s \mid \omega\left\{q_{1} \vee q_{2}\right\}} \quad[\mathrm{D} 2] \quad \frac{p_{1} \operatorname{co} q_{1} \text { in } F \mid \omega \quad p_{2} \operatorname{co} q_{2} \text { in } F \mid \omega}{\left(p_{1} \vee p_{2}\right) \operatorname{co}\left(q_{1} \vee q_{2}\right) \text { in } F \mid \omega} \\
& \text { [D3] } \frac{p \operatorname{co} q \text { in } F \mid \omega}{(p \wedge r) \cos (q \wedge r) \operatorname{in} F \mid \omega} \dagger \quad[\text { D4 }] \quad \frac{(p \wedge X=e) \operatorname{co} q \text { in } F \mid \omega}{p \cos q \operatorname{in} F \mid \omega} \ddagger \\
& \text { [D5] } \frac{p \wedge m=X \text { leads }(p \wedge m<X) \vee q \text { in } F \mid \omega}{p \text { leads } q \text { in } F \mid \omega} \\
& \text { [D6] } \frac{p_{1} \operatorname{co} q_{1} \text { in } F_{1} \mid \omega_{1} \quad p_{2} \operatorname{co} q_{2} \text { in } F_{2} \mid \omega_{2}}{\left(p_{1} \wedge p_{2}\right) \operatorname{co}\left(q_{1} \wedge q_{2}\right) \text { in } F_{1} \| F_{2} \mid \omega_{1} \cup \omega_{2}} \sharp \\
& \dagger \operatorname{var}(r) \cap \omega=\emptyset \\
& \ddagger \quad x \notin(\operatorname{var}(p) \cup \operatorname{var}(e) \cup \operatorname{var}(q) \cup \operatorname{var}(F)) \\
& \text { } \downarrow m \text { is well-founded with respect to }< \\
& \# \operatorname{var}\left(q_{1}\right) \cap \omega_{2}=\emptyset \wedge \operatorname{var}\left(q_{2}\right) \cap \omega_{1}=\emptyset
\end{aligned}
$$

Figure 6: Additional inference rules

because the synchronous combination does not interleave states in a computation. As a consequence, co properties are enough to define progress properties (see rule C10), and we may drop the concept of transient predicates [20] and existentially quantified triples [8]. However, to preserve the semantics of leads, we add rule C9.

To make the DSYNC logic useful in practice, the basic set of rules of Figure 4 must be extended with several derived rules. Figure 6 shows some of these rules. Derived rules include adaption rules for command triples which are lifted to properties and transition triples. For instance, rule D1 for command triples originates rule D2 for co properties. Rules D3 and D4 are similarly derived from rules for command triples.

Other derived inference rules are inherited (with little changes) from UNITY. For instance, D 5 is an induction rule for leads properties that comes from UNITY. Finally, some derived rules are specific to synchronous transitions. For instance, D6 describes the synchronous combination of co properties. This last group of derived rules is essential because they reflect basic aspects of synchronous transitions.

To prove a derived rule where the premises and the conclusion are formulas of the same kind, we frequently need induction on the proof length. This is the case in rules D2, D3, and D4, where both the premises and the conclusion are co properties. To derive a rule for transition triples from a rule for command triples, the basic rule B2 gives the base case, and the basic rules B1, B3, and B4 give the induction steps.

Likewise, to derive a rule for co properties from a rule for transition properties, the basic rule $\mathrm{C} 4$ gives the base case, and the basic rules $\mathrm{C} 3, \mathrm{C} 6$, and $\mathrm{C} 7$ give the induction steps. Before this proof, we need to expand the second premise in rules $\mathrm{C} 6$ and $\mathrm{C} 7$ by the definition of inv given by $\mathrm{C} 5$, exposing a hidden premise on co. We deal with leads properties in a similar way. It must be observed that the basic adaption rules B1, C1, C3, and C8 in Figure 4 cannot be derived from A1 using this technique; they must be basic rules.

We claim that the DSYNC logic is sound, i.e., rules in Figure 4 only generate true 
formulas. To justify this claim, we consider each layer separately, greatly simplifying the soundness proof. The soundness of the bottom layer comes from the Hoare logic [1], and the soundness of the middle and top layers follows from the semantics of the DSYNC transition system, and from the definitions of triples and properties.

To illustrate the soundness proof for the middle layer, we sketch a proof that rule B4 is sound. Let $\sigma$ be a state where $\widehat{\sigma}(p)=\operatorname{true.~Assume~the~premises~of~B4~are~true.~}$ From this assumption, it follows that the component transitions $t_{1}$ and $t_{2}$ terminate when their computations start in $\sigma$, the resulting states satisfy $q$, and they only write to $\omega_{1}$ and $\omega_{2}$. Rule B4 is sound if all computations of $t_{1} \| t_{2}$ starting in $\sigma$ also terminate, the resulting states also satisfy $q$, and they only write to $\omega_{1} \cup \omega_{2}$. We demonstrate this statement next.

Figure 2 defines the semantics of $t_{1} \| t_{2}$ using rules S9 and S10. We analyze rule S9 first. From the assumption above, it follows that $t_{i} \mid \omega_{i}^{\prime} \triangleright \sigma \stackrel{\operatorname{trn}}{\longrightarrow} \sigma_{i}$, and $\widehat{\sigma}_{i}(q)=\operatorname{true}$, and $\omega_{i}^{\prime} \subseteq \omega_{i}$. Therefore, according to S9, the execution of $t_{1} \| t_{2}$ beginning in $\sigma$ terminates producing $\sigma^{\prime}$ given by $\sigma \oplus\left(\sigma_{1} \downarrow \omega_{1}^{\prime}\right) \oplus\left(\sigma_{2} \downarrow \omega_{2}^{\prime}\right)$, and writing to $\omega_{1}^{\prime} \cup \omega_{2}^{\prime}$. Since $\omega_{i}^{\prime} \subseteq \omega_{i}$, we already get that the synchronous combination only writes to $\omega_{1} \cup \omega_{2}$. It remains to show that $q$ holds in $\sigma^{\prime}$.

When two states agree on the value of all variables of an expression, this expression has the same value in both states. We claim that $q$ holds in $\sigma^{\prime}$ because it holds in $\sigma_{1}$, and $\sigma^{\prime}$ and $\sigma_{1}$ agree on the value of all variables of $q$. Let $x$ be a variable in $\operatorname{var}(q)$. From the proviso of $\mathrm{B} 4$ and from $\omega_{i}^{\prime} \subseteq \omega_{i}$, we get that either $x \in \omega_{1}^{\prime}$ and $x \in \omega_{2}^{\prime}$, or $x \notin \omega_{1}^{\prime}$ and $x \notin \omega_{2}^{\prime}$.

Let $\varphi$ and $\varphi^{\prime}$ be states, let $\nu$ be a write-set, and let $y$ be a variable. The definitions of $\oplus$ and $\downarrow$ entail the propositions below:

$[\mathrm{P} 1]$ if $y \in Z$, then $\left(\varphi \oplus\left(\varphi^{\prime} \downarrow Z\right)\right)(y)=\varphi^{\prime}(y)$;

$[\mathrm{P} 2]$ if $y \notin Z$, then $\left(\varphi \oplus\left(\varphi^{\prime} \downarrow Z\right)\right)(y)=\varphi(y)$;

[P3] if $y \notin \nu$ and $t \mid \nu \triangleright \varphi \stackrel{\operatorname{trn}}{\longrightarrow} \varphi^{\prime}$, then $\varphi^{\prime}(y)=\varphi(y)$.

First, assume $x \in \omega_{1}^{\prime}$. From P1 and the definition of $\sigma^{\prime}$, it follows that $\sigma^{\prime}(x)=\sigma_{1}(x)$. Alternatively, assume $x \notin \omega_{1}^{\prime}$. In this case, we also have that $x \notin \omega_{2}^{\prime}$. From P2 and the definition of $\sigma^{\prime}$, we get that $\sigma^{\prime}(x)=\sigma(x)$. But P 3 and the definition of $\sigma_{1}$ ensure that $\sigma_{1}(x)=\sigma(x)$ too. So, we also get that $\sigma^{\prime}(x)=\sigma_{1}(x)$.

Since there are no other cases to consider, we get that $\sigma^{\prime}$ and $\sigma_{1}$ agree on the value of all variables in $\operatorname{var}(q)$, and $q$ holds in $\sigma^{\prime}$. A symmetric argument shows that the resulting state of $t_{1} \| t_{2}$ according to $\mathrm{S} 10$ also satisfies $q$. Therefore, rule B4 is sound. Similar arguments show that all rules in Figure 4 are sound.

It is worth to observe that the soundness proof of the top layer is independent and simpler than the soundness proof of UNITY and avoids some foundation problems on the UNITY logic [21]. This is a consequence of some differences between DSYNC and UNITY, such as the synchronous combinator and the absence of infinitary rules and stuttering steps. We still have no completeness results for DSYNC logic, but we are working on this.

The layered organization of this logic is an important design decision because it allows for a separation of concerns. Results about the bottom layer are inherited from the standard Hoare logic. In the middle layer, we analyze the synchronous combinator 
without concerns on the notation for elementary transitions, or on the temporal logic. The top layer deals with reachable states and complete program computations. Although this layer is based on the UNITY logic, there are evidences that we could switch to other similar logic such as TLA or the Manna and Pnueli logic. Such change does not affect the previous layers, and the new temporal logic could equally inherit the results from other layers.

\section{Pragmatics and Extensions}

DSYNC inherits many specification and verification techniques from its base formalisms (UNITY and the Hoare logic), but new techniques must be developed to cope with the specificities of DSYNC. To prove a property or triple, we usually begin at the conclusion and proceed backwards to the given premises, using the proof rules to break formulas into sub-formulas. The overall proof organization reflects the program structure and the organization of its computations. As the synchronous combinator is the main control structure in DSYNC, the rules for this construction strongly influence the organization of proofs in this formalism.

Rules B3 and B4 break a triple over a synchronous combination into sub-triples over its component transitions, but the proviso in these rules impose restrictions on the sub-triples and their write-sets. To satisfy these restrictions, we usually formulate subtriples that reflect the contribution of each transition to the desired result. For instance, let $t_{1}$ and $t_{2}$ be (if $x \geq 0$ then $y:=x$ else skip) and (if $x<0$ then $y:=-x$ else skip), respectively. Below, we list a proof for property $x=X$ co $y=a b s(X)$ in $t_{1} \| t_{2} \mid\{x\}$ :

(1) $x=X \wedge x \geq 0$ co $y=X \wedge X \geq 0$ in $t_{1} \mid\{x\}$

(2) $x \geq 0$ co true in $t_{2} \mid \emptyset$

(3) $x=X \wedge x \geq 0$ co $y=X \wedge X \geq 0$ in $t_{1} \| t_{2} \mid\{x\}$

(4) $x=X \wedge x<0$ co $y=-X \wedge X<0$ in $t_{1} \| t_{2} \mid\{x\}$

(5) $(x=X \wedge x \geq 0) \vee(x=X \wedge x<0)$ co $(y=X \wedge X \geq 0) \vee(y=-X \wedge X<0)$ in $t_{1} \| t_{2} \mid\{x\}$

(6) $x=X$ co $y=\operatorname{abs}(X)$ in $t_{1} \| t_{2} \mid\{x\}$
$\mathrm{A} 1-6, \mathrm{~B} 2$, and $\mathrm{C} 4$

$\mathrm{A} 1-6, \mathrm{~B} 2$, and $\mathrm{C} 4$

D6 in $(1,2)$, and $\mathrm{C} 3$ similar to $(1-3)$

D2 in $(3,4)$

C3 in (5)

Upper-case variables stand for rigid variables. These variables are not modified through assignment, preserving their value between states. We use these variables in properties to state relations between the initial and final value of program variables. Using these variables, the properties in lines 1 and 2 above describe the effect (contribution) of the elementary transitions on the final value of variables when $x \geq 0$. Next, line 3 describes the synchronous combination of these transitions. Due to the dynamically built write-sets, we may show that there is no conflict between $t_{1}$ and $t_{2}$ when the considered states satisfy $x \geq 0$. As a complement, line 4 deals with the case when $x<0$. The desired property follows from the disjunction of both cases.

This example illustrates a general proof tactic. Initially, we split a property into sub-properties corresponding to computation paths sharing the same write-sets. For instance, we split line 5 into lines 3 and 4 corresponding to the branches of the selection statements. Next, we splip a property and its write-set into the component transitions. For instance, we splip line 3 into lines 1 and 2, also splitting the associated write-set. 


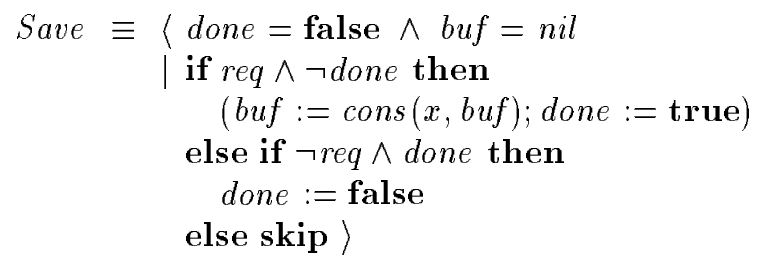

Figure 7: Program saving $x$ to buf

This proof tactics reflects the program computation and is reasonably intuitive to the system designer.

This proof tactic is very elementary and widely applicable. Another common tactic uses rigid variables and rules D3 and D4 to restrict properties to specific states. However, as we proceed to more complex systems and specifications, we need more sophisticate proof techniques. Elsewhere [23], we present a catalog of basic specification and verification techniques for DSYNC which may be applied in many situations. Like the tactics above, they depend on the program structure, on the organization of its computations, and on the kind of property that must be proved. Broadly, these techniques reflect the programmer's knowledge on the system he or she is designing, and they are easy to select and apply. Therefore (except for some hard cases), usually it is quite easy to find a proof in DSYNC.

As a more complex example, consider program Save in Figure 7 . Constant nil is the empty list, and cons $(x, l)$ appends element $x$ to the beginning of list $l$. Whenever variable req indicates a request, program Save pushes the value of $x$ in the list buf, and indicates through done that the operation is finished. When the request is removed, done is reset. This is an elementary example of open system, a system that interacts with an environment which is not described together with the system. The proper operation of an open system usually depends on some assumptions on the environment.

To deal with environment assumptions, we use conditional properties. A conditional property is an "if-then" rule, where the premises and the conclusion are ordinary properties. A statement about an open system $F$ is represented as a conditional property where the conclusion refers to $F \| G$ and the premises describe $G$. The program $G$ is an undefined program representing the environment, and its properties are assumptions on the environment. The property in the conclusion describes the open system $F$ through a description of the interaction between $F$ and $G$.

As an example, consider the conditional property below:

$$
\frac{r e q \wedge x=X \wedge \neg \text { done co } r e q \wedge x=X \text { in } G \mid r e q, x, \omega}{r e q \wedge x=X \wedge \text { buf }=B \text { leads buf }=\text { cons }(X, B) \wedge \text { done in Save } \| G \mid \text { done, buf }, \text { req, }, w, \omega}
$$

The property in the premise indicates that the environment does not change the value of variables $x$ and req when there is an unattended request, and the property in the conclusion indicates that $x$ eventually will be stored in buf, and done eventually will be turned on. Therefore, this conditional property indicates that Save will store $x$ in buf under the assumption that the environment keeps the value of $x$ stable. 
To fully specify program Save, we need two additional properties:

$$
\begin{gathered}
r e q \wedge \text { done } \wedge \text { buf }=B \text { co } \text { buf }=B \text { in Save } \mid \text { done, buf } \\
\neg r e q \wedge \text { buf }=B \text { co buf }=B \wedge \neg \text { done in Save } \mid \text { done }, \text { buf }
\end{gathered}
$$

The first property describes what happens after a request is granted. In this case, the value of buf does not change. The second property deals with the situation where there is no request. In this case, the value of buf also does not change and additionally done remains off. These properties are not conditional because they do not depend on any assumptions on the environment behavior.

The set of properties above illustrates a common specification pattern in DSYNC, which describes a system module as an open system that should wait for operation requests. There is a set of associated proof techniques similar to the ones described earlier in this section, which aid in the verification of these properties. Elsewhere [23], we analyze other common specification patterns and proof techniques. Although proofs in the DSYNC logic cannot be fully automated, we can use these specification and verification techniques to offer a quite extensive partially automated support for proof development. To realize this goal, we plan to embed DSYNC into a programmable theorem prover such as HOL.

Open systems are fundamental for modular system development. Each component in a library may be regarded as an open system to be plugged into a complete system latter on. Conditional properties allow for the verification of properties of a component based on properties of other components. It also allows for the derivation of system properties from component properties. These tasks do not depend on any knowledge on the actual components. This means that a component may be replaced by other, as long as both present the same properties. Since any property may be a premise, we may place arbitrary restrictions on the environment. Other approaches (e.g., model-checkers [9]) impose severe limits on environment restrictions, thus limiting their applicability.

DSYNC may be extended in several directions. Simple but very useful additions are generic parameters and regular structures. Generic parameters are unspecified constants. They usually stand for the amount of some system resource (size of a vector, number of available network connections, and so on). DSYNC represents a generic parameter as a rigid variable. A regular structure is a set of transitions following a common syntactical pattern parametrized on an argument $I$. To represent the regular structure $t_{0}\left\|t_{1}\right\| \ldots\left\|t_{E-2}\right\| t_{E-1}$, DSYNC adopts the notation $\left\langle 0 \leq I<E: t_{I}\right\rangle$. Generic parameters and regular structures allow for very compact system descriptions. For instance, assume $N$ is a generic parameter. Let $a$ be a vector. A program $F_{a}$ which sets the first $N$ elements of $a$ to zero can then be written simply as $\langle 0 \leq I<N: a[I]:=0\rangle$.

In [25], we study a treatment for generic parameters and regular structures in a static version of DSYNC. The same approach may be adopted for the present version of DSYNC. However, in the case of regular structures, its usefulness is limited by the lack of indexed variables in write-sets. We are working on this problem. 


\section{Related Work}

The features of DSYNC discussed along this paper are essential for the verification of synchronous systems. However, they are not present in other formalisms based on a first-order liner-time temporal logic. UNITY $[8,20]$ is a typical formalism based on a transition system and a temporal logic. It is simple and flexible, and it was applied to a large set of examples. UNITY includes a synchronous combinator, but its logic does not include rules to prove properties about it. Therefore, this combinator becomes a syntactic sugar to describe complex transitions. The UNITY notation restricts transitions to conditional multiple assignments, so it syntactically forbids non-terminating transitions. The multiple assignments must not generate write-conflicts, but UNITY does not specify when such conflicts happen, or how we check their absence.

Other formalisms based on a linear-time temporal logic present similar problems. To verify a system, the Manna and Pnueli logic [17, 18] and TLA [16] include methods to represent several programming constructions as logical formulas. However, these methods do not deal with synchronous systems or write-conflicts. ST [30] is a specialpurpose formalism that includes a static method to handle synchronous transitions. However, its method only handles a restricted subset of properties and systems, and it is harder to apply because it generates an exponential number of proof subgoals.

Besides the transition systems and temporal logics, DSYNC is also related to research on the semantics and verification of VHDL $[5,10,11]$. The works on this field differ on the subset of VHDL they handle, and on the formalism they use. Some works $[3,32]$ cover large subsets of this language, but the resulting semantics are too complex, and are not appropriate for the formal verification of actual designs. Other works are oriented towards the formal verification of $\operatorname{VHDL}[4,7,31]$, but they only cover restricted subsets of the language. The static version of DSYNC addresses a similarly restricted subset of VHDL [24]. Additionally, it also handles some features that other semantics usually ignore (e.g., generic parameters and regular structures), and can be easily extended to the dynamic conflict resolution method. DSYNC also includes a general and clear analysis of the synchronous combinator, while most works mix such analysis with the study of other aspects of VHDL. This approach may lead to very subtle errors [6].

We may employ DSYNC in other verification tasks beyond VHDL. Evolving algebras $[12,13,14]$ are a specification formalism which is applied to the description of programming languages and computational systems. Since it adopts a synchronous computation model, we may verify properties of an evolving algebra using the DSY NC logic. We are developing a version of DSYNC tailored to evolving algebras, what amounts to adapting the DSYNC logic to the conflict resolution method of evolving algebras, and to indexed write-sets. We are aware of only one other logic for evolving algebras [28], and we believe the DSYNC logic is more expressive and easier to use than this logic.

DSYNC may be also applied to the verification of synchronous programming lan-

guages such as Esterel [2]. These languages adopt the same computation model of DSYNC, although usually they also include a notion of real time measured in some actual time unit. Through the introduction of some restrictions in these languages, programs are represented as a finite automaton, which allows for efficient verification 
techniques [15]. DSYNC is a more general formalism, which does not impose restrictions on programs. We believe we may use the DSYNC logic as a complement to standard verification methods for synchronous programming languages, which we apply in systems or description violating the restrictions above. To fulfill this goal, we are exploring a model of real time for DSYNC.

Nowadays, there is a lot of work on formal verification employing finite modelcheckers, automata, and similar techniques [19]. Such approaches have been successfully applied on many fields, including hardware description languages and synchronous programming languages $[9,15,27]$. They are fully automated and quite effective. However, despite advances in the field, these techniques are not appropriate to all situations. They do not deal with first-order (non-propositional) specifications, and are not appropriate for the verification of modular systems, regularly structured systems, parametric descriptions, dynamically detect conflicts, or data-intensive designs. It seems that a linear-time temporal logic such as DSYNC is a nice complement to these techniques, since it easily handles those hard situations. Some works explore this complementary nature [4], and we plan to explore this path in some future developments of DSYNC.

\section{Last Remarks}

DSYNC is composed of a transition system and an associated linear-time temporal logic. The DSYNC transition system adopts the synchronous computation model, includes a dynamic method to solve write-conflicts, represents transitions as possibly non-terminating imperative commands, and may handle generic parameters and regular structures. The DSYNC logic is designed to verify properties of such transition systems. Proofs in this logic are compositional, meaning that properties of a system may be derived from properties of its components without refering to the actual definition of components. Therefore, DSYNC allows for modular system development and verification. The DSYNC logic is organized in layers, allowing for a separation of concerns, and simplifying the formalism development and its soundness proof. There is a catalog of elementary specification and verification techniques in DSYNC which covers several types of systems and properties. Some of these techniques were inherited (possibly with changes) from other formalisms, while some are specific to DSYNC.

These features of DSYNC are essential for the verification of synchronous systems. However, as the previous section discussed, they are not present in other formalisms based on a first-order liner-time temporal logic. This work was motivated by a wish to apply a first-order linear-time temporal logic to the verification of VHDL designs. Such logics have been successfully applied to several application fields, but they deal with asynchronous systems mostly. DSYNC resulted from adapting UNITY, a wellknown formalism based on a linear-time temporal logic, to the verification of synchronous systems with dynamic conflict resolution. Besides VHDL, the resulting formalism may be applied to the verification of other hardware description languages, to synchronous programming languages, and to specification formalisms.

There are many aspects of DSYNC which need to be further developed. We would like to extend the DSYNC programming notation with named cells with formal parameters, and we need to show that the DSYNC logic is complete. We are adapting DSYNC 
to evolving algebras, and we plan to develop some automated support to DSYNC. We also plan to further study the complementary link between DSYNC and some automated verification techniques such as model-checkers.

To summarize, DSYNC allows for the application of a first-order linear-time temporal logic to the verification of synchronous systems. It may be employed in several application fields, it is quite general and easy to use, and it is complementary to other more restricted and automated verification methods.

\section{References}

[1] K. R. Apt and E.-R. Olderog. Verification of Sequential and Concurrent Programs. Springer-Verlag, 1991.

[2] G. Berry. The Esterel v5 language primer. Ecole des Mines and INRIA, 1997.

[3] E. Börger, U. Glässer, and W. Müller. The semantics of behavioral VHDL'93 descriptions. In EURO-VHDL'94, pages 500-5, 1994.

[4] D. D. Borrione et al. Formal verification of VHDL descriptions in the Prevail environment. IEEE Design \& Test of Computers, 9(2):42-55, 1992.

[5] D. Borrione, editor. Special issue on VHDL semantics. Formal Methods in System Design, 7(1/2), 1995.

[6] P. T. Breuer et al. A simple denotational semantics, proof theory and validation condition generator for unit-delay VHDL. Formal Methods in System Design, $7(1 / 2): 27-52,1995$.

[7] P. T. Breuer et al. A refinement calculus for the synthesis of verified hardware descriptions in VHDL. ACM TOPLAS, 19(4):586-616, 1997.

[8] K. M. Chandy and J. Misra. Parallel Program Design. Addison-Wesley, 1988.

[9] D. Déharbe et al. The CV model-checker. In FMCAD'98. Springer-Verlag, 1998.

[10] C. Delgado Kloos and P. T. Breuer, editors. Formal Semantics for VHDL. Kluwer, 1995.

[11] C. Delgado Kloos and W. Damm, editors. Practical Formal Methods for Hardware Design. Springer-Verlag, 1997.

[12] Y. Gurevich. Evolving algebras: An attempt to discover semantics. University of Michigan, 1994.

[13] Y. Gurevich. Evolving algebras 1993: Lipari guide. In Specification and Validation Methods. Oxford University, 1995.

[14] Y. Gurevich. May 1997 draft of the ASM guide. University of Michigan, 1997. 
[15] L. Jagadeesan et al. Safety property verification of ESTEREL programs and applications to telecommunications software. In $C A V-95,1995$.

[16] L. Lamport. The temporal logic of actions. ACM TOPLAS, 16(3):872-923, 1994.

[17] Z. Manna and A. Pnueli. The Temporal Logic of Reactive and Concurrent Systems: Specification. Springer-Verlag, 1992.

[18] Z. Manna and A. Pnueli. Temporal Verification of Reactive Systems: Safety. Springer-Verlag, 1995.

[19] K. C. McMillan. Symbolic Model Checking. Kluwer, Boston, 1993.

[20] J. Misra. A logic for concurrent programming. University of Texas at Austin, 1994.

[21] P. Päppinghaus. On the logic of UNITY. Theoretical Computer Science, 139:27-67, 1995.

[22] D. L. Perry. VHDL. McGraw-Hill, 1991.

[23] V. M. Rodrigues. Transições Síncronas, Lógica Temporal e VHDL. Tese de doutorado, CPGCC, UFRGS, 1998.

[24] V. M. Rodrigues and F. R. Wagner. A temporal logic for data-flow VHDL. In $X I$ Symposium on Integrated Circuits and System Design, pages 91-94. IEEE Computer Society, 1998.

[25] V. M. Rodrigues and F. R. Wagner. A logic for synchronous transitions. In SBLP'99. Sociedade Brasileira de Computação, 1999.

[26] F. B. Schneider. On Concurrent Programming. Springer-Verlag, 1997.

[27] A. Scholz et al. The FORMAT model checker. In Practical Formal Methods for Hardware Design, pages 175-183. Springer-Verlag, 1997.

[28] A. Schönegge. Extending dynamic logic for reasoning about evolving algebras. Technical report, Universität Karlsruhe, 1995.

[29] A. U. Shankar. An introduction to assertional reasoning for concurrent systems. ACM Computing Surveys, 25(3):225-262, 1993.

[30] J. Staunstrup. A Formal Approach to Hardware Design. Kluwer, 1994.

[31] J. P. V. Tassel. Femto-VHDL: The Semantics of a Subset of VHDL and its Embedding in the HOL Proof Assistant. PhD thesis, University of Cambridge, 1992.

[32] P. A. Wilsey et al. A model of VHDL for the analysis, transformation, and optimization of digital system designs. In $C H D L-95$, pages $611-6,1995$. 